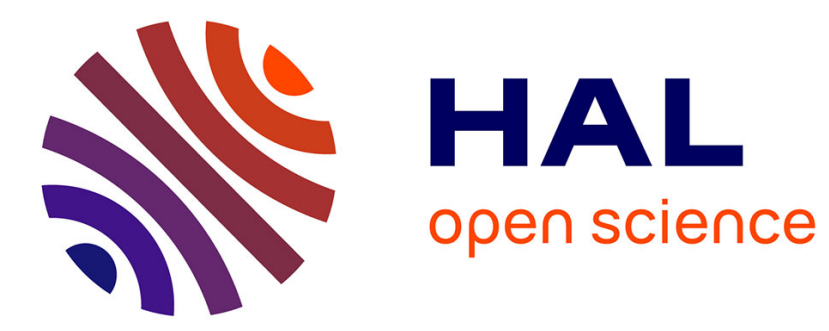

\title{
Vers la construction d'une expertise européenne en matière de coopération pénale? Spécialisation et légitimation des professionnels de la coopération judiciaire.
}

Antoine Mégie

\section{To cite this version:}

Antoine Mégie. Vers la construction d'une expertise européenne en matière de coopération pénale? Spécialisation et légitimation des professionnels de la coopération judiciaire.. Droit et Société, 2010, 74, pp.129-150. halshs-00757150

\author{
HAL Id: halshs-00757150 \\ https://shs.hal.science/halshs-00757150
}

Submitted on 26 Nov 2012

HAL is a multi-disciplinary open access archive for the deposit and dissemination of scientific research documents, whether they are published or not. The documents may come from teaching and research institutions in France or abroad, or from public or private research centers.
L'archive ouverte pluridisciplinaire HAL, est destinée au dépôt et à la diffusion de documents scientifiques de niveau recherche, publiés ou non, émanant des établissements d'enseignement et de recherche français ou étrangers, des laboratoires publics ou privés. 


\title{
Vers la construction d'une expertise européenne en matière de coopération pénale ? Spécialisation et légitimation des professionnels de la coopération judiciaire.
}

\author{
Antoine Mégie \\ Université de Rouen
}

Vers la construction d'une expertise européenne en matière de coopération pénale ? Spécialisation et légitimation des professionnels de la coopération judiciaire.

Cet article entend démontrer l'importance d'analyser les professionnels de la coopération judiciaire afin de comprendre les orientations politiques et normatives qui définissent l'institutionnalisation de l'espace pénal européen. En s'appuyant sur une sociologie des magistrats et hauts fonctionnaires des ministères nationaux de la Justice et des institutions européennes, nous analyserons les modalités et ressources qui conduisent ces acteurs à devenir de véritables experts des affaires européennes. La compréhension des logiques d'actions individuelles et collectives de ces professionnels conduit à interroger la forme et le contenu de l'expertise en matière de coopération pénale européenne qui s'impose au début des années 2000.

Coopération européenne - justice pénale - expertise - professionnels du droit - sociologie du droit

To the definition of an European Judicial Expertise in Criminal Matters? Specialization and Legitimization of the Professionals of the Judicial Cooperation.

This paper aims the importance of analysing the professionals of the judicial cooperation to characterize the normative and political transformations within the European judicial space. It draws on sociological analysis of the judges and Civil servants of the National and European administrations, this article focus on the different forms of European specialization in criminal matters. The understanding of the logics of action which define these professionals enables to give a meaning to the normative form of the European judicial expertise produced at the dawn of the 21 st century. 
Depuis le début des années 1990, la coopération judiciaire est devenue un secteur singulier de la construction européenne à travers la multiplication des dispositifs d'entraide tels que : les magistrats Schengen, les magistrats de liaison, le Réseau Judiciaire Européen (RJE), l'unité Eurojust ou encore la procédure d'extradition européenne plus connue sous le nom de mandat d'arrêt européen. L'ambition était dans le cas des magistrats Schengen, des magistrats de liaison et du RJE, qui ont été respectivement les premiers instruments adoptés, de faciliter les transmissions d'informations et de procédures entre les autorités judiciaires nationales grâce à la création de postes spécifiques en charge des ce travail au sein des juridictions nationales (magistrats Schengen et RJE), ou à l'étranger dans les ambassades (magistrats de liaison). La création de l'unité européenne Eurojust et du mandat d'arrêt européen s'inscrivent dans cette même logique de renforcement des mécanismes d'échanges d'informations, de procédures mais aussi de personnes ${ }^{1}$.

L'observation des principales mobilisations qui ont structuré directement ou indirectement l'institutionnalisation de la coopération judiciaire permet de comprendre l'importance qu'une partie des professionnels du droit occupe dans la formulation des problèmes à résoudre et du savoir faire juridique mais aussi politique à mobiliser (juristes, magistrats et hauts fonctionnaires des administrations nationales et européennes) ${ }^{2}$. Les perceptions et croyances de ces acteurs vont incarner les représentations dominantes et légitimes, au point de devenir autonomes et de se diffuser aux autres sphères $^{3}$.

Cet article propose une analyse de ces professionnels qui, au cours des années 1990, s'imposent comme les spécialistes de la coopération judiciaire européenne. Du fait de ce statut d'expert, ces magistrats et hauts fonctionnaires des ministères nationaux de la Justice constituent de véritables

\footnotetext{
1. Eurojust : Première unité européenne exclusivement consacrée à la coopération judiciaire, Eurojust constitue un office multilatéral regroupant un représentant des instances judiciaires de chaque État de l'UE. Dans une logique de coopération verticale entre l'échelon européen et national, sa mission est de faciliter les échanges entre les juridictions nationales dans le cadre d'affaires criminelles transnationales. L'intervention des procureurs d'Eurojust se focalise donc essentiellement sur la coordination de la procédure pénale.

Le Mandat d'arrêt européen: Le mandat d'arrêt européen $(E A W)$ représente une procédure spécifique d'extradition entre les États membres de l'UE. Introduisant de nombreuses innovations juridiques vis-à-vis du régime classique de l'extradition, l'EAW marque la mise en œuvre du principe européen de reconnaissance mutuelle des décisions juridiques dans un nouveau champ du droit. Obligeant les juridictions européennes de l'UE à reconnaître les jugements des instances étrangères, ce principe, qui ne s'appliquait qu'en matière commerciale et civile, devient un axe central dans l'évolution de la coopération pénale.

2. Antoine MEGIE (dir), "Arrêter et juger en Europe : genèse, luttes et enjeux de la coopération pénale », Cultures et Conflits, $\mathrm{n}^{\circ} 62$, Paris, l'Harmattan, Printemps 2006.

${ }^{3}$. Yves SUREL, «L'intégration européenne vue par l'approche cognitive et normative des politiques publiques », Revue française de science politique, vol. 50, $\mathrm{n}^{\circ}$ 2, avril 2000, p. 241.
} 
entrepreneurs $d u d r o i t^{4}$. En effet, grâce à leurs positions institutionnelles et leurs connaissances, ces derniers participent directement à la gestion de l'agenda politique et anticipent les possibles blocages ou accords des Chancelleries nationales qui restent dans le domaine intergouvernemental de la sécurité européenne les principaux décideurs. Dans ces conditions, ces acteurs pèsent directement dans la formulation des énoncés politiques et des dispositifs d'entraide. Leur étude conduit à s'intéresser de facto aux problématiques relatives à la professionnalisation à l'Europe ${ }^{5}$ ainsi qu'à celles concernant la fabrication d'une nouvelle expertise dans le domaine de la coopération pénale, au sens d'un ensemble de savoirs et de savoirs faire judiciaires influençant le contenu de l'action publique ${ }^{6}$.

S'appuyant sur un travail de recherche effectué entre 2001 et 2006 dans le cadre d'une thèse, notre étude se concentre sur une vingtaine de professionnels français, anglais, belges, néerlandais, espagnols et italiens ${ }^{7}$. Ces acteurs qui participent à l'institutionnalisation de la coopération judiciaire occupent des postes au sein de leurs administrations nationales ou bien dans les services du Conseil de l'UE et de la Commission européenne. Si la plupart de ces acteurs sont des magistrats, la spécificité de chaque institution nationale en charge des questions judiciaires implique la présence d'autres types d'acteurs que nous qualifions de hauts fonctionnaires. De la sorte, nous proposons de compléter les travaux consacrés aux membres du Secrétariat Générale du Conseil de l’UE (SGCE) et des professionnels du droit ayant participé à l'institutionnalisation de la coopération judiciaire européenne $^{8}$. En effet, tout en intégrant les représentants du SGCE dans notre analyse, nous nous intéressons également aux autres professionnels travaillant à l'échelle nationale et européenne. Or ce sont les orientations de cet ensemble d'acteurs qui vont être à l'origine de l'adoption des différents dispositifs d'entraide en particulier l'unité Eurojust et le mandat d'arrêt européen alors que les promoteurs de l'Appel de Genève et du projet Corpus Juris en faveur d'un procureur européen vont être écartés du processus décisionnel.

4. Au sens développé par Howard Becker concernant «les entrepreneurs moraux»: Howard BECKER, Outsiders, The Free Press of Glencoe, 1963, Trad. Paris, Editions Métailié, Paris, 1985.

5. Didier GEORGAKAKIS (dir), Les Métiers de l'Europe politique : acteurs et professionnalisations de l'Union européenne, Strasbourg, Presses universitaires de Strasbourg, 2002.

${ }^{6}$. Voir notamment: Laurence DUMOULIN, « De l'impact des experts judiciaires sur la fabrique de la justice : d'une justice imposée à une justice négociée ? », dans Laurence DUMOULIN, Stéphane LA BRANCHE, Cécile ROBERT, Philippe WARIN (dir), Le recours aux experts : raisons et usages politiques, Grenoble, Presses universitaires de Grenoble, coll. « Symposium », 2005.

7. Afin de respecter l'anonymat - souvent demandé - des personnes interviewées, nous ne préciserons que la nationalité et/ou la fonction des personnes citées. Par ailleurs, si les acteurs français constituent le contingent le plus important, les données recueillis sur ces derniers demeurent représentatives au regard de celles rassemblées sur les membres des autres Etats.

${ }^{8}$. Michel MANGENOT, Hélène MICHEL, Natacha PARIS, Une Europe de la justice : mobilisation contre la "criminalité organisée" et institutionnalisation d'un espace judiciaire pénal européen (1996-2001), Paris, Mission de recherche Droit et Justice, 2004. 
Ces magistrats et hauts fonctionnaires, qui alimentent la construction des normes de coopération, représentent un ensemble hétérogène du fait de positions et de ressources institutionnelles diverses. $\mathrm{Au}$ carrefour de plusieurs domaines, les problématiques de la coopération judiciaire constituent un point de convergence pour ces acteurs qui vont s'intégrer progressivement au cœur du champ des professionnels de la sécurité ${ }^{9}$ Le principal danger est alors de construire un groupe superficiellement homogène et autonome à travers l'énonciation de tendances générales. Dans ses analyses sur la construction d'un champ transnational en matière commerciale et de la défense de l'environnement, Yves Dezalay souligne un tel risque. En s'intéressant aux juristes qui participent à la redéfinition de ces espaces sociaux, l'auteur définit les acteurs professionnels comme des intermédiaires, servant de courtiers entre le monde social et le monde du travail ${ }^{10}$. Refusant de parler d'un champ autonome à proprement parler Yves Dezalay, évoque un «espace carrefour où s'affrontent des agents - militaires, universitaires, bureaucrates et managers - dont les stratégies et les ressources proviennent pour l'essentiel d'autres champs structurés en fonction d'histoires nationales spécifiques ${ }^{11} \gg$. Une telle approche des professionnels du droit et de leur participation aux processus d'internationalisation et d'européanisation se retrouve également dans les travaux relatifs aux origines de la définition d'un ordre politique international et européen ${ }^{12}$. Dans ces différents cas, les auteurs montrent comment des coalitions de juristes réussissent à imposer aux politiques le fait que le droit peut faire office de science réaliste et efficace pour une gouvernance internationale ou européenne. Dès lors, l'expertise en matière juridique et les acteurs qui la construisent se trouvent au centre des dispositifs. Cependant, comme le soulignent, là aussi, fortement les auteurs, la communauté de spécialistes reste largement éclatée et hétérogène avec une multitude de positions d'expertise. Ces remarques trouvent tous leurs intérêts dans le secteur de la coopération judiciaire. En effet, l'activité des acteurs que nous étudions a pour conséquence d'introduire la coopération judiciaire et plus largement le droit en tant qu' élément essentiel pour la légitimation du domaine européen de la sécurité qui s'était structuré à l'origine exclusivement sur les problématiques de coopération policière et d'échanges d'informations entre services répressifs.

9. Didier BIGO, «La mondialisation de l'(in)sécurité ? Réflexions sur le champ des professionnels de la gestion des inquiétudes et analytique de la transnationalisation des processus d'(in)sécurisation », Cultures \& Conflits, Paris, l'Harmattan, $\mathrm{n}^{\circ}$ 58, 2005, p. 53-100.

10. Yves DEZALAY, Garth BRYANT, The Internationalization of Palace Wars: Lawyers, Economists, and the Contest to Transform Latin American States, Chicago. Ill, London : University of Chicago Press , 2002; Antonin COHEN, Antoine VAUCHEZ (dir.), La Constitution européenne : élites, mobilisations, votes, Bruxelles, éditions de l'Université de Bruxelles, 2007, p. 271-291.

${ }^{11}$. Yves DEZALAY, «De la défense de l'environnement au développement durable. L'émergence d'un champ d'expertise des politiques européennes ", Actes de la recherche en sciences sociales, nº166/167, 2007, p. 68.

${ }^{12}$. Guillaume SACRISTE, Antoine VAUCHEZ, «Les «bons offices» du droit international : la constitution d'une autorité non politique dans le concert diplomatique des années 1920 », Critique internationale, Paris, $\mathrm{n}^{\circ} 25,2005$, p. 101-117. 
Pour autant et malgré des logiques d'actions qui se structurent encore de manière importante dans le cadre national, on observe une unité sociale entre ces acteurs qui se traduit par la production et le partage d'un ensemble de croyances, de pratiques et de savoirs faire concernant la coopération judiciaire. On peut, dès lors, se demander pourquoi et comment ces professionnels réussissent à produire une expertise relativement homogène qui leur permet de participer et de peser sur les décisions politiques en matière pénale?

Afin de résoudre cette énigme sociologique, il convient d'appréhender les principales logiques d'actions à l'œuvre grâce à l'analyse des clivages et des régularités autour desquels ils s'organisent. La spécialisation aux questions européennes ne doit pas être considérée comme un phénomène magique et naturel de l'européanisation, mais au contraire comme le résultat d'investissements individuels. Cette perspective nous conduit à travailler sur les dynamiques de spécialisation grâce auxquelles ces acteurs s'imposent au centre du jeu institutionnel. Il s'agit ainsi de définir les conditions d'entrée et les modalités à partir desquelles certains individus réussissent à faire autorité de façon durable et, à l'inverse, comment d'autres sont relégués. Le principal intérêt est de démontrer que l'évolution du droit ne s'effectue pas simplement par un glissement jurisprudentiel, mais par une transformation des représentations, des cadres législatifs et des pratiques due, en partie, à la façon dont certains professionnels se positionnent en tant qu'intermédiaire entre les niveaux de gouvernements grâce à un multipositionnement qui leur permet d'intervenir dans les négociations et l'adoption des dispositifs européens de coopération.

Quelles sont les ressources mobilisées pour accéder et être présent de façon pérenne et légitime dans l'espace communautaire ? Dans quelle mesure le fait de détenir des savoirs et savoirs faireparticuliers donne-t-il à ces acteurs une autonomie leur permettant de participer individuellement et collectivement au processus politique européen?

Dans le but de répondre à ces questionnements et d'ouvrir véritablement la boîte noire du processus de production des normes pénales européennes, nous définirons, dans un premier temps, les mécanismes qui conduisent certains magistrats et hauts fonctionnaires à s'investir dans la coopération judiciaire européenne ainsi que les modalités grâce auxquelles ils acquièrent une position d'autorité. Par la suite, nous montrerons comment l'analyse des trajectoires de spécialisation et des ressources accumulées permet de comprendre le type d'expertise qui se construit et s'impose au cours des années 1990. Nous pourrons ainsi donner du sens aux orientations politiques et normatives qui définissent l'institutionnalisation de l'espace judiciaire européen. 


\section{Les voies de la spécialisation dans la coopération judiciaire européenne}

Afin d'interroger les clivages et logiques d'action autour desquels s'organisent les professionnels de la coopération judiciaire, nous présenterons tout d'abord de manière précise une série de trajectoires observées qui nous permettront de montrer comment la spécialisation dans le domaine européen de la justice pénale se construit grâce à une circulation entre les différents niveaux de pouvoir (européens et nationaux principalement). Cela nous permettra, par la suite, de mettre en lumière les principales voies par lesquelles certains magistrats et hauts fonctionnaires ont investi ce secteur. Une telle étude offre la possibilité de comprendre les effets que les trajectoires professionnelles ont sur les prises de positions discursives et institutionnelles des acteurs.

\section{1 Elément central de la spécialisation : la circulation entre les niveaux de gouvernance}

L'institutionnalisation durant les années 1990 d'un domaine européen en matière pénale a eu pour effet de multiplier les postes en charge de ces questions à l'échelle européenne et nationale. A cette époque, les principaux postes concernant spécifiquement ce secteur se situent au niveau, des Représentations permanentes nationales à Bruxelles (RP), des groupes de travail et comités ad hoc du Conseil des ministres européens, des fonctions de magistrats Schengen, de magistrats de liaison et de membres du Réseau judiciaire européen (RJE). Les postes au sein des autorités centrales et fédérales des États membres ont également une grande importance. Du fait de la singularité politique et administrative de chaque pays de l'UE, on constate de profondes diversités dans l'organisation interne de ces autorités. C'est la raison pour laquelle les fonctions concernant les affaires de coopération européenne en matière judiciaire sont très différentes, selon les pays, tant en ce qui concerne leurs prérogatives que la manière dont elles sont valorisées. Face à cette diversité, il convient de focaliser l'étude sur les trajectoires d'une partie des professionnels qui réussissent à valoriser leur travail pratique, juridique ou politique dans ce secteur afin d'être nommés à différents postes en charge de ces questions.

Les personnes recrutées lors de la création, en 1994, de la direction générale «Justice et Affaires Intérieures » (JAI) du Secrétariat général du Conseil (SGCE), sont à ce titre exemplaire. Comme l'a démontré Michel Mangenot, la prise en compte du parcours de ces acteurs, pour la plupart 
hauts fonctionnaires, est d'autant plus intéressante que ces derniers vont être au cœur de la formulation juridique et politique de l'unité Eurojust ${ }^{13}$ et du mandat d'arrêt européen.

- Le responsable des questions de coopération pénale au ministère luxembourgeois depuis trente ans, et cofondateur du groupe TREVI, est nommé directeur général.

- Le poste de directeur juridique est occupé par un fonctionnaire ayant vingt ans d'expérience sur ces questions au sein du ministère néerlandais de la Justice. Étroitement lié au pouvoir politique, ce haut fonctionnaire batave est également l'un des rédacteurs de la convention Schengen.

- Au sein de cette DG, une direction « coopération judiciaire et policière » est mise en place, avec à sa tête l'ancien chef de cabinet du ministre belge de la Justice depuis 1989. À ce titre, il fut associé à la proposition de 1993 des autorités belges relative au CIREJUD. À l'instar de son collègue néerlandais, ce haut fonctionnaire a également participé à la construction de l'accord Schengen.

- Enfin, le chef de la division « coopération judiciaire » est un magistrat suédois qui a travaillé dix ans au Conseil de l'Europe sur ces questions.

Le fait que ces hauts fonctionnaires ou magistrats aient contribué pour une grande partie d'entre eux à la construction de l'accord Schengen, revêt une importance particulière dans la manière dont ces derniers penseront et définiront, par la suite, les normes judiciaires de coopération qui doivent, selon eux, être mises en place.

Il est courant de voir des professionnels ayant occupé un poste dans leur Chancellerie nationale sur les questions européennes durant les années 1980, être recrutés au niveau européen soit en tant que représentant national (RP nationale, groupes ad hoc du Conseil des ministres européens, magistrats de liaison, magistrats Schengen) soit au sein des institutions européennes et communautaires (SGCI, Commission européenne, Office Européen de lutte anti-fraude (OLAF)).

$\mathrm{Au}$ niveau français, la création de services administratifs spécialisés dans l'entraide pénale européenne au début des années 1990 s'accompagne d'une forte mobilité entre les différentes fonctions. En effet, quelques professionnels, en l'espèce des magistrats, vont «circuler» entre les services de la Chancellerie en charge des questions de coopération européenne : Service des affaires européennes et internationale (SAEI) ; services diplomatiques auprès du Premier Ministre qui assurent les négociations à l'échelle européenne ${ }^{14}$; services de la Représentation permanente (RP) à Bruxelles ; et les dispositifs diplomatiques et judiciaires comme le poste de magistrat de liaison.

\footnotetext{
13. Michel, MANGENOT, «Jeux européens et innovation institutionnelle. Les logiques de création d'Eurojust (1996-2004) », Cultures \& Conflits, Paris, l'Harmattan, n62, Printemps 2006, pp 43-62.

14. Notamment le Secrétariat général du comité interministériel appelé également SGCI. Depuis octobre 2005 le SGCI est devenu le Secrétariat général des affaires européennes (SGAE).
} 
Dans le courant des années 1990 et au début des années 2000, plusieurs magistrats évoluent ainsi au niveau de ces différentes autorités et jouent un rôle important dans les processus politiques et juridiques qui aboutiront à la création d'Eurojust et du mandat d'arrêt européen.

- Ainsi, cette magistrate qui après s'être occupée au sein du SAEI de la mise en place des premiers dispositifs d'entraide (magistrats Schengen, magistrats de liaison) est devenue magistrate de liaison à l'étranger.

- Cette voie est suivie quelques années plus tard par son remplaçant au sein du SAEI. Celui-ci fut chef du service JAI de la RP française à Bruxelles, après avoir été directeur de la formation continue à l’École de la Magistrature, chef du SAEI puis secrétaire général adjoint au SGCI.

- Le magistrat qui inaugure le premier poste de conseiller JAI à la RP française à Bruxelles en 1997, devient magistrat de liaison, après avoir occupé la direction du groupe du Conseil des ministres européens «coopération judiciaire pénale » durant la présidence française de l'Union en 2000 à l'époque de la négociation du projet Eurojust.

Un autre parcours de spécialisation qui passe également par le SAEI est intéressant à mentionner eu égard à son exemplarité et à la place occupée par ce magistrat dans les différents systèmes, notamment en tant que magistrat Schengen. Ayant commencé sa carrière au sein des juridictions nationales, ce dernier devient au début des années 1990, Chef de la mission justice du service des affaires européennes et internationales auprès de la Direction centrale de la Police judiciaire. Par la suite, ce magistrat est détaché au ministère de la Défense pour devenir Chef du bureau du droit européen. À la fin des années 1990 et aux débuts des années 2000, il occupe le poste de Chef du bureau du droit pénal européen et international du ministère de la Justice. Enfin, depuis cinq ans, ce magistrat français est « expert détaché » auprès de la Commission européenne.

À travers ces trajectoires, on peut évoquer la construction, en France, d'une «filière de spécialisation » avec pour point central le SAEI en tant que niveau essentiel d'intégration. Ce type de circulation n'est pas spécifique au cas français. D'autres trajectoires similaires peuvent pareillement être évoquées : le magistrat de liaison néerlandais, présent jusqu'en 2006 en France, a auparavant travaillé sur ces questions dans sa Chancellerie, puis deux ans au sein du SGCE à la fin des années 1990 où il a participé à la rédaction du projet Eurojust.

Par ailleurs, cette circulation entre les différents postes en charges des questions européennes peut également amener certains magistrats ou hauts fonctionnaires à faire carrière en dehors de leur administration nationale. L'institutionnalisation d'un domaine communautaire en matière judiciaire offre l'occasion à certains acteurs d'intégrer les postes au sein des organes du Conseil de l'UE, de la direction générale JLS de la Commission européenne ou encore de l'Office Européen de lutte anti 
fraude (OLAF). L'exemple des membres du SGCI constitue bien sûr un premier exemple important. Exemplaire de ce type de trajectoire, le directeur de la DG «coopération judiciaire et policière » de SGCI est devenu en 2007, le coordinateur européen anti-terroriste.

A un autre niveau institutionnel, certains acteurs décident de présenter leur candidature aux concours communautaires. Dans le cas d'un recrutement, ces derniers démissionnent alors de leur administration nationale.

- Ainsi, cette magistrate française qui, après avoir travaillé à l'École de la magistrature française sur les questions internationales et européennes, a participé à la rédaction du projet de mandat d'arrêt européen en tant qu'experte française détachée à la Commission européenne, pour enfin être recrutée au sein de la DG JAI à la direction « coopération pénale ».

- Autre exemple, ce procureur néerlandais qui, après avoir été en poste à Rotterdam, fut nommé expert national dans différents groupes européens en charge de la lutte contre la criminalité financière. Comme il le souligne lui-même, c'est grâce à ses expériences nationales et européennes qu'il a pu être recruté aux services judiciaires de l'OLAF au début des années 2000.

Pour certains professionnels, les postes dans les institutions communautaires constituent, du fait de leurs modalités de recrutement, un moyen de contourner les obstacles qu'ils rencontrent au sein de leur administration nationale.

- Symptomatique de ces configurations plusieurs fois perçues, le cas de ce magistrat qui, après avoir travaillé à la rédaction du projet Eurojust et avoir été écarté du poste de représentant national, a décidé d'intégrer les services de l'OLAF.

On constate donc une circulation des acteurs entre les différents postes européens et nationaux. Ce phénomène a pour effet de réduire le nombre de professionnels spécialisés dans le domaine de la coopération judiciaire. Ainsi, à partir du milieu des années 1990, un groupe relativement restreint de magistrats et de hauts fonctionnaires aux parcours et expériences ancrés dans les affaires internationales deviennent progressivement les premiers spécialistes de la coopération judiciaire européenne. Le fait de se positionner en tant que précurseurs et détenteurs de savoirs et de savoirs faire spécifiques et nouveaux va permettre à ces professionnels de se mouvoir dans différentes fonctions du domaine de la coopération judiciaire.

\section{2 Des conditions d'accès diverses entre expérience de terrain et investissement institutionnel.}


Si l'on étudie de manière comparative les parcours des différents acteurs rencontrés, il semble possible de dégager deux voies principalement empruntées pour accéder à la spécialisation judiciaire européenne et par la suite être nommé à une fonction en charge de cette matière. L'entrée dans la coopération judiciaire se structure ainsi soit par l'occupation d'un poste au sein des institutions nationales compétentes sur ces questions soit par l'expérience de terrain. A partir de ces premières implications dans les questions de coopération transnationale des filières se mettent en place dans lesquelles il devient possible de faire carrière, avec l'émergence de postes gagnant en visibilité. Des trajectoires-types se dessinent, incitant certains fonctionnaires ou magistrats à s'investir dans la matière européenne: "À cette époque, les questions internationales étaient un domaine où l'on pouvait rapidement avoir des responsabilités intéressantes. On pouvait se retrouver très tôt avec des fonctions et des compétences importantes si l'on arrivait à intégrer certains postes ${ }^{15}{ }^{\prime}$.

Dans le cas de l'accès via la voie institutionnelle, les acteurs commencent à construire leur expertise au sein des diverses autorités politiques centrales compétentes en matière de justice pénale en s'occupant des questions de formulation des normes pénales en matière de coopération transnationale et de leur mise en œuvre juridique. Dans ce cas et en fonction des Etats, ces professionnels ne sont pas exclusivement des magistrats. C'est le cas, par exemple, du responsable des questions de coopération pénale au sein du Ministère Belge de la justice qui a participé activement à la formulation du projet Eurojust et de la décision cadre du mandat d'arrêt européen.

Pour ces professionnels, le travail au sein de ces Chancelleries ou ministères en charge des questions européennes porte essentiellement sur la dimension technique et diplomatique de l'entraide. Ces acteurs interviennent donc plus spécifiquement dans le registre juridique, en matière de transposition des normes et décisions européennes, ou politique lorsque les services des ministères doivent produire des notes en amont des négociations ou sommets européens. Membres des institutions politiques de leurs pays, la nomination et la durée du mandat de ces hauts fonctionnaires ou magistrats peuvent, en fonction des États, être largement dépendante de l'alternance politique. Dans ces conditions et comme nous le préciserons par la suite, la question des ressources d'ordre politique et/ou syndical peut prendre une grande importance en fonction des pays.

Une partie des parcours évoqués précédemment nous montre que ce type d'accès aux affaires européennes se retrouve pour un nombre conséquent de professionnels. Une autre voie d'accès à la spécialisation, que l'on peut qualifier d'accès par le terrain, est également observable et mérite d'être évoqué.

\footnotetext{
${ }^{15}$. Entretien réalisé avec un magistrat français, février 2003.
} 
Concernant cette entrée dans le domaine de la coopération européenne, la question soulevée par Antoine Vauchez, dans son analyse de la magistrature italienne, de « l'inégalité d'accès » aux matières judiciaires en raison du temps de la procédure et de la répartition géographique des affaires, apparaît des plus pertinentes ${ }^{16}$. En effet, dans le cas de la coopération judiciaire européenne, de par la nature des infractions qui nécessitent une telle collaboration, tous les juges des États membres de l'UE ne sont pas confrontés à ce domaine, malgré un élargissement croissant des infractions qualifiées de la sorte. Dans la pratique, ces affaires représentent une minorité des cas traités par les autorités judiciaires nationales. Une telle dimension permet de donner du sens à la première explication formulée par une grande majorité des acteurs eux-mêmes, selon laquelle leur investissement dans les questions européennes est dû au hasard : «Ce qui a déterminé mon orientation, c'est aussi le hasard des permanences. Il se trouve que les grandes affaires de stupéfiants internationaux sont tombées sur mes permanences. C'est le hasard des choses qui a fait ça ${ }^{17}{ } »$.

En fait, ce que les acteurs qualifient de «hasard» peut être interprété comme le résultat de l'inégalité d'accès qui existe entre les magistrats sur les affaires européennes. L'ouverture au domaine de l'entraide pénale dépend de la capacité des juges à prolonger leur expérience dans ce type de contentieux, en accumulant le nombre de dossiers traités en la matière. Plusieurs facteurs permettent de comprendre que certains magistrats sont confrontés plus que d'autres à ce type spécifique d'enquêtes judiciaires.

La compétence géographique des magistrats est une première explication. Un grand nombre de personnes occupant un poste dans la coopération européenne ont travaillé dans des juridictions qui, en raison de leur situation géographique, regroupaient le traitement d'affaires transnationales. Certains magistrats compétents dans des zones transfrontalières, ou bien dans des zones incluant des grands ports, ont ainsi été confrontés de manière directe à la question de l'entraide. Du fait d'un contexte géographique particulier, ces juges sont très fréquemment en charge des contentieux impliquant une entraide bilatérale avec les pays frontaliers. Afin de mener à bien leur procédure, les magistrats doivent connaître les règles de coopération et donc les principales caractéristiques du système judicaire du pays voisin. Un tel phénomène d'apprentissage est également observable pour les forces de police, notamment entre la France, l'Allemagne et la Belgique ${ }^{18}$.

L'institutionnalisation, à partir du début des années 1990, des dispositifs de coopération tel que les magistrats Schengen ou, par la suite, le Réseau judiciaire européen a également conduit certains

\footnotetext{
${ }^{16}$. Antoine VAUCHEZ, L'institution judiciaire remotivée : le processus d'institutionnalisation d'une "nouvelle justice" en Italie : 1960-2000, Paris, LGDJ, coll. « Droit et Société », 2004.

17. Entretien réalisé avec un magistrat français, juin 2003.

${ }^{18}$. Pour une étude des logiques de coopération transfrontalière voir : Azilis MAGUER, Les frontières intérieures Schengen: Dilemmes et stratégies de la coopération policière et douanière franco-allemande, Max Planck Institut, éd. Iuscrim, 2004.
} 
magistrats à travailler, au sein de leur juridiction nationale, de manière constante sur les procédures d'entraide européenne. La création de ces postes spécialisés dans l'envoi et la réception des procédures de coopération a servi de point d'entrée pour des magistrats qui ultérieurement vont se spécialiser dans le domaine européen.

La nature des infractions traitées dans le cadre national constitue une autre explication. Une partie des magistrats rencontrés évoquent ainsi une spécialisation dans des affaires de «criminalité transnationale organisée» (CTO). L'expérience dans la lutte contre les phénomènes de CTO représente un des principaux dénominateurs communs à une grande partie des magistrats qui s'engagent dans les affaires européennes durant les années 1990. Les magistrats en charge de ces questions, notamment dans des pays où le système judiciaire permet une spécialisation en la matière, vont être les premiers à véritablement s'intéresser à l'enjeu européen, dans une stratégie essentiellement opérationnelle. Le poste de magistrats de liaison en Italie fut ainsi crée afin de renforcer l'entraide bilatérale sur la question de la « criminalité organisée ». Le fait que la coopération judiciaire ait été définie comme enjeu politique européen à travers la lutte contre les phénomènes de CTO doit être mis en relation avec un tel processus de reconversion. Il est intéressant de remarquer, une fois encore, que ce phénomène existe très largement dans le cas des forces de police ${ }^{19}$.

En raison des facteurs géographiques, organisationnels et/ou du type d'affaires traitées, certains magistrats nationaux se trouvent de façon plus constante en charge de procédures à dimension transnationale ${ }^{20}$. La confrontation régulière à ces contentieux conduit une partie de ces professionnels à accumuler des expériences en la matière, ce qui par la suite leur permet d'être recruté ou nommé à des fonctions consacrées spécifiquement à la coopération européenne.

Le début de la spécialisation aux questions de coopération judiciaire européenne se fait donc de deux façons : soit par le traitement judiciaire des contentieux transnationaux, soit dans le cadre de fonctions au sein des autorités nationales. Ces deux types d'entrée ne sont pas sans conséquence sur la façon dont ces professionnels vont, par la suite, se définir individuellement et collectivement. La prise en compte de l'évolution des carrières permet de comprendre comment pour certains professionnels les expériences pratiques et/ou techniques s'accumulent, leur permettant, de construire leur expertise et leur légitimité.

\section{Une expertise produit des logiques de spécialisation, de légitimation et de socialisation}

19. Didier BIGO, Polices en réseaux, Presses de Sciences Po, 1996.

20. Wanda DE LEMOS DE CAPPELER, « La transnationalisation du champ pénal : réflexions sur les mutations de crise et de contrôle », Droit et société, n 35, 1997, p. 61-77. 
Les professionnels qui s'engagent dans l'entraide européenne ont en commun d'avoir une connaissance singulière d'un secteur en cours d'institutionnalisation. Si toutes ces propriétés leur permettent de se positionner avec succès sur le terrain européen et de l'ériger en spécialité à part entière, cela ne signifie pas que l'ensemble de ces professionnels participent de la même manière à la construction des dispositifs de coopération. Se pose, dès lors, la question de la division du travail entre les membres du groupe. Le poids des affiliations nationales, et des parcours professionnels apparaît comme un facteur déterminant faisant de ce groupe un ensemble d'acteurs hétérogènes. La compréhension de cette dimension nous renvoie directement à la problématique de la légitimité, et par là même, à la question concernant les raisons pour lesquelles certains réussissent à imposer leur autorité d'expert durablement dans un domaine politique européen en pleine évolution.

\subsection{La fabrication du statut d'expert entre accumulation des ressources et multipositionnement social}

Les changements organisationnels intervenus à partir du milieu des années 1990 dans les administrations nationales et européennes conduisent à une valorisation institutionnelle et symbolique de la spécialisation européenne en matière de justice pénale ${ }^{21}$. Dans une telle configuration, des ressources qui ne sont pas des attributs classiques pour faire une «carrière » dans le domaine de la Justice, se transforment en atouts dès lors qu'il s'agit de trouver des représentants susceptibles de s'occuper des questions de coopération. Tout comme les professionnels participant à la transnationalisation du champ commercial ou de la défense de l'environnement, les professionnels de la coopération judiciaire doivent pour s'imposer dans l'espace européen mobiliser certaines ressources spécifiques à celui-ci. En raison des parcours personnels et des cadres nationaux dans lesquels leurs carrières s'inscrivent, la variabilité des ressources entre les acteurs apparaît comme une caractéristique importante.

Les professionnels qui ne peuvent s'appuyer sur des connaissances linguistiques de bon niveau sont rapidement relégués par les autres acteurs au rang d' "amateurs". Cette dimension est régulièrement soulignée par l'ensemble des acteurs. Le témoignage de cette magistrate qui fut chargée de recruter des membres du RJE atteste de l'importance accordée à une telle ressource : «Je me suis retrouvé à une réunion aux côtés d'un collègue qui ne parlait pas bien l'anglais. Il n'a pas ouvert la

21. Violaine ROUSSEL, «Les magistrats français, des cause lawyers malgré eux ?», Politix, vol. 16, $\mathrm{n}^{\circ} 62$, 2003, p. 93-113. 
bouche durant la réunion, sa présence n'avait donc aucun intérêt ${ }^{22} »$. Dans cette perspective, l'appartenance à une double culture et/ou le fait d'avoir effectué des études ou suivi des formations à l'étranger, sont régulièrement mis en avant comme autant d'atouts. La connaissance d'une langue particulière représente pour certains postes et certains pays le facteur principal de la nomination

Une autre ressource apparaît également décisive dans le cas de la coopération européenne. En étroite relation avec la question de la diffusion des énoncés et des discours, celle-ci concerne la possibilité pour les professionnels de s'investir dans des projets éditoriaux consacrés aux questions européennes. La multiplication exponentielle de la littérature institutionnelle sur le secteur européen de la sécurité depuis une dizaine d'années se trouve au centre du processus de légitimation d'une partie de ces professionnels de la coopération pénale. L'étude des livres consacrés à ce sujet à travers la fonction institutionnelle de leurs contributeurs est très instructive.

Les acteurs qui contribuent à la construction d'Eurojust et du mandat d'arrêt européen participent pour la quasi-totalité d'entre eux à la rédaction d'ouvrages qui sont publiés à cette époque. L'exemple le plus emblématique est sans contexte celui des membres du SGCE. En effet, le directeur de la direction « coopération judiciaire et policière » et le chef de la division « coopération judiciaire » vont être à l'origine de nombreux écrits académiques en tant que principal auteur ou directeur de publication. Des professionnels français, italiens et belges vont également contribuer à ces publications en tant que magistrat de liaison, membre d'une RP nationale ou représentant national au sein d'un groupe ad hoc du Conseil des ministres européens.

Ces ressources éditoriales sont importantes car elles jouent un rôle majeur dans la construction du statut d'expert. En effet, l'écriture d'un article permet à son auteur de gagner en visibilité et d'asseoir son expertise notamment vis-à-vis des autres professionnels. Cette légitimation passe par une valorisation de l'expérience pratique et juridique à travers l'écrit et la diffusion de celui-ci dans les différentes enceintes qu'elles soient politiques ou académiques, telles que le Parlement européen, les services de la Commission européenne et du Conseil de l'UE ou les universités engagées dans les programmes de recherche européens.

La possibilité de mobiliser de telles ressources est d'autant plus importante que généralement ces écrits s'inscrivent dans une démarche de proposition et de défense d'une orientation précise en termes de normes de coopération à mettre en place. Les clivages politiques et juridiques concernant les instruments de coopération sont au centre de ces logiques éditoriales qui se développent à partir des années 1990 dans le domaine de la coopération judiciaire européenne. Vont ainsi s'opposer par rapports officiels et livres interposés les promoteurs du projet Corpus Juris en faveur d'une

\footnotetext{
${ }^{22}$. Entretien réalisé avec magistrat néerlandais travaillant dans la coopération judiciaire, février 2003.
} 
harmonisation des législations pénales, les magistrats à l'origine de l'Appel de Genève souhaitant un renforcement de la coopération et de l'indépendance de la justice, et les magistrats et hauts fonctionnaires défendant une démarche favorable à la mise en place de règles minimum de coopération dans une logique avant tout opérationnelle et concrète. La possibilité de multiplier les supports éditoriaux et de les faire circuler au sein des Chancelleries nationales prend dans ces conditions une importance cruciale.

En outre, les moyens éditoriaux sont souvent associés à d'autres ressources qui, elles aussi, concourent largement à la construction du statut d'expert. Il s'agit en l'espèce de l'activité de professeurs ou d'intervenants au sein d'universités ou d'écoles spécialisées (Instituts européens ou École de formation pour la magistrature). Ces interventions permettent à certains professionnels d'être à l'origine des programmes européens d'expertise financés par les autorités nationales ou communautaires.

À partir de notre recherche empirique, ces ressources paraissent être au cœur du processus de légitimation puisque la très grande majorité des hauts fonctionnaires et magistrats, ayant contribué à la mise en forme juridique et politique d'Eurojust et du mandat d'arrêt européen, les détiennent de manière plus ou moins équivalente. Comme nous le verrons par la suite, le fait de participer à ces activités permet, par ailleurs, à ces professionnels de se reconnaître entre eux et de sanctionner leur légitimité d'expert.

En raison de l'importance des cadres nationaux dans les trajectoires personnelles, d'autres ressources peuvent également jouer un rôle central. Dans certains cas, il semble que les ressources politiques et/ou syndicales via l'appartenance à un parti et/ou à un syndicat constituent un atout majeur dans la nomination au niveau européen. Le passage d'une partie des membres du SGCE par des cabinets ministériels apparaît comme un exemple significatif. Il en va de même de la participation active à la création d'Eurojust d'un des premiers magistrats de liaison français qui, proche des réseaux socialistes, fut conseiller auprès de la ministre de la Justice durant la présidence française de l'UE en 2000. Dans le cas italien, les ressources syndicales et politiques apparaissent également essentielles eu égard à l'importance que cette dimension occupe dans le système judiciaire transalpin ${ }^{23}$. Un magistrat italien devenu l'un des premiers et principaux représentants transalpins dans le domaine de la coopération judiciaire européenne bénéficiait auparavant d'une très forte reconnaissance pour son action dans la lutte contre la mafia et le terrorisme à laquelle il associait des ressources politiques et syndicales importantes en tant qu'ancien dirigeant syndical et militant politique actif.

23. Antoine VAUCHEZ, Op. cit, 2004. 
Il reste tout de même difficile, dans le cas d'un groupe aussi éclaté et hétérogène que celui que nous étudions, d'établir une typologie précise des attributs à mobiliser pour être nommé et faire autorité dans le secteur de la coopération judiciaire européenne. Face à cette difficulté, il est pertinent de formuler la question des ressources de façon particulière en interrogeant les conditions qui font que certains acteurs réussissent à œuvre à la formulation et à la mise en œuvre des politiques pénales européennes.

L'investissement dans le domaine européen s'accompagne d'un impératif d'apprentissage et de transformations relativement fort du savoir faire professionnel. C'est pourquoi, les professionnels doivent gérer leur passé, en termes d'identité professionnelle, d'image publique et de routines professionnelles. Certains parcours et attributs relativement "marginaux» peuvent alors, sous la pression de l'européanisation, se convertir en ressources face aux nouveaux cadres d'action. Les professionnels qui parviennent le mieux à s'adapter à ces ajustements sont ceux qui réussissent à accumuler le plus de ressources leur permettant de devenir des acteurs multipositionnés. Cette multipositionnalité, qui les fait se situer au carrefour des champs des pouvoirs nationaux ${ }^{24}$ grâce à leur insertion dans différents réseaux, constitue une hypothèse explicative forte. Dans un domaine au carrefour du champ politique, judiciaire et académique, la capacité pour les acteurs à se mouvoir entre les divers cercles représente un moyen de s'imposer comme expert et surtout de participer durablement au jeu européen.

La très grande majorité des professionnels qui s'imposent comme les experts incontournables de la coopération judiciaire européenne au milieu des années 1990 parvient à cumuler un poste institutionnel, un travail éditorial et/ou d'enseignement et différents engagements syndicaux ou politiques qu'ils soient au niveau européen ou national. Ces jeux croisés de reconnaissance et de cumul des positions ont pour conséquence de réserver à un nombre encore plus restreint de spécialistes les postes importants lors des processus d'adoption et de mise en œuvre des politiques instituant les différents dispositifs de coopération comme Eurojust et le mandat d'arrêt européen. Ceux qui n'arrivent pas à se construire en acteur multipositionné sont écartés du jeu et ne peuvent durablement être impliqué dans la formulation des normes de coopération. Ce multipositionnement revêt également une place non négligeable dans la manière dont ces professionnels interagissent et construisent de façon collective et individuelle leur approche générale de la coopération judiciaire européenne.

\subsection{Les clivages professionnels comme sanction d'une légitimité politique et judiciaire}

\footnotetext{
${ }^{24}$. Antonin COHEN, Yves DEZALAY, Dominique MARCHETTI, « Introduction : Esprits d'État, entrepreneurs d'Europe », Actes de la recherche en sciences sociales, ${ }^{\circ}$ 166-167, 2007, p. 4-13.
} 
Loin de représenter un ensemble homogène, les professionnels investis dans la coopération judiciaire européenne se définissent autour de différentes lignes clivages qu'ils construisent euxmêmes afin de sanctionner leur légitimité.

Les clivages renvoyant aux appartenances nationales sont extrêmement présents. L'appartenance à un système de Common Law est souvent considérée comme une «déviance » par rapport à l'idée d'intégration européenne en matière judiciaire. La dimension nationale représente sans aucun doute la première ligne de clivages entre les différents membres du groupe. Les formations et cultures juridiques pèsent sur la manière dont les acteurs se définissent. L'opposition schématique système accusatoire/système inquisitoire est souvent présentée comme un facteur explicatif déterminant. Cependant, cette logique de différenciation en fonction des nationalités ne constitue pas l'unique dynamique de différenciation.

Une autre ligne de clivage, fortement liée aux types d'apprentissages initiaux, est également largement mise en avant par les acteurs afin de sanctionner leur propre légitimité. En effet, en déterminant les ressources de l'engagement et, par la suite, une partie des logiques de formation empirique, les apprentissages initiaux influencent la manière dont chaque professionnel se définit et valorise son approche de la coopération judiciaire ${ }^{25}$. Le fait de pouvoir mettre en valeur ses connaissances institutionnelles ou opérationnelles constitue pour les personnes rencontrées un moyen de légitimer leur action et leur position. On constate la construction d'une forte différenciation entre les acteurs se qualifiant de « juges de terrain » et les professionnels en charge des questions politiques. Selon la position et la nature de la spécialisation dont se réclame chaque acteur, les connaissances diplomatiques ou pratiques sont plus ou moins valorisées : "Comme je le disais au début, avec le développement des activités internationales que la personne soit au départ un magistrat ou pas, cela est important. Un magistrat qui a fait du terrain et qui ensuite siège dans une RP constitue un atout ${ }^{26} »$.

Il est intéressant de remarquer que le clivage entre professionnels politiques et professionnels de terrain ne concerne pas uniquement la division magistrats/hauts fonctionnaires, puisqu'il est également utilisé régulièrement au sein même du groupe des magistrats, comme l'atteste ce témoignage : «Certains magistrats n'ont pas beaucoup d'expérience de terrain. Le fait d'être resté deux ans dans une juridiction ne signifie pas que l'on connaît la réalité pratique. Certains sont dans les institutions

\footnotetext{
${ }^{25}$. Paul BOUFFARTIGUE, «Introduction », in Yvette LUCAS, Claude DUBAR (dir.), Genèse et dynamique des groupes professionnels, Lille, Presses Universitaires de Lille, 1994, p. 327-339.

26. Entretien réalisé avec un magistrat français membre de la Représentation Permanente à Bruxelles et ancien magistrat de liaison, février 2001.
} 
politiques depuis plus de dix ans. Donc le terrain et les problèmes pratiques, ils ne le connaissent pas forcément très bien ou seulement par les rapports qu'ils lisent ${ }^{27} »$.

Les professionnels qui ont commencé à se spécialiser à travers le traitement d'affaires judiciaires à dimension européenne présentent ainsi leur connaissance du terrain comme un atout fondamental de leur expertise. Dans cette perspective, la capacité à combiner connaissances pratiques et techniques est définie comme leur valeur ajoutée.

Dans une dynamique de légitimation et en fonction de la position et du travail de chacun, les acteurs valorisent donc leurs compétences «pratiques » et/ou « politiques », comme autant de moyens de s'affirmer vis-à-vis des autres professionnels. Ainsi, la grande majorité des hauts fonctionnaires rencontrés défendent l'idée qu'en raison de la nature des négociations et du jeu institutionnel européen, leur statut exclusivement politique et administratif constitue un élément de distinction important par rapport aux autres professionnels du jeu que sont les magistrats: "Je ne suis pas magistrat. Je fais de la négociation depuis vingt ans. Alors c'est très bien de faire appel aux magistrats parce qu'ils ont des connaissances de terrain. Mais l'inconvénient, c'est qu'il y a moins de distance par rapport à son système. Or si l'on veut avancer dans la construction européenne, il faut pouvoir dire, on a fait comme ça jusqu'à présent mais demain on fera autrement. Quand il y a des coupures majeures à faire, pour moi c'est mieux d'avoir comme interlocuteur des gens qui n'ont pas ce lien. Il y a une tendance à défendre des positions qui correspondent à certaines cultures de travail ${ }^{28} »$.

La communauté des professionnels, investie dans la coopération judiciaire européenne dès le milieu des années 1990, se définit et se structure à partir de nombreuses lignes de clivage concernant aussi bien la nationalité, la position socioprofessionnelle, la voie d'accès à la spécialisation ou encore le niveau d'intervention dans l'action publique. Pourtant, dans le même temps, face à une telle hétérogénéité, ce groupe se construit autour d'un certain nombre de représentations et de perceptions communes en particulier sur la question de ce que doit être la coopération judiciaire européenne dans sa forme juridique et politique. L'environnement social dans lequel ces derniers évoluent occupe alors une place explicative importante.

\subsection{Le partage d'une expertise «pragmatique » et opérationnelle de la coopération pénale}

\footnotetext{
27 . Entretien réalisé avec magistrat italien, février 2003.

${ }^{28}$. Entretien avec un haut fonctionnaire du ministère de la Justice belge, février 2003.
} 
Le resserrement du nombre d'acteurs, en raison des logiques de spécialisation et de légitimation, favorise la mise en place de liens sociaux entre ces professionnels à travers leur travail quotidien et leurs autres activités éditoriales ou syndicales, par exemple. La centralité et la concentration des institutions à Bruxelles constituent un facteur important dans la construction de ces rapports. De véritables relations de proximité se développent autour des multiples réseaux dans lesquels chaque professionnel est inséré. De nombreux acteurs entretiennent ainsi des liens d'amitié, voire ont des conjoints qui travaillent également dans les affaires européennes au niveau de la justice mais aussi des forces de police ${ }^{29}$.

Ces dynamiques sociales qui irriguent de manière classique le processus européen ${ }^{30}$, sont par ailleurs renforcées par le partage entre ces professionnels de ce qu'ils qualifient d'une expérience commune. Soulignée précédemment, la représentation qu'ils se font d'eux en termes de "pionniers » et «d'inventeurs » en matière de coopération européenne durant les années 1990, constitue un paramètre essentiel dans la manière dont ces professionnels définissent leur approche de façon individuelle mais aussi collective.

En raison de ces différentes dynamiques, on constate donc, malgré les nombreux clivages et singularités des trajectoires personnelles, une homogénéité des catégories de perceptions et de représentations ${ }^{31}$.

Le premier type de représentations communes réside dans le fait de considérer la coopération européenne comme une solution politique et institutionnelle essentielle pour la réalisation de l'Union européenne. Plusieurs éléments en ce sens sont évoqués par l'ensemble des acteurs rencontrés dont celui faisant de la coopération entre les Etats-membres le moyen le plus approprié face aux phénomènes criminels. Dans cette perspective, les représentations sociales identifiant la criminalité organisée et le terrorisme en termes de menace transnationale se trouvent au cœur des logiques discursives. Ces représentations sont d'ailleurs un des principaux points communs avec les autres professionnels du champ de la sécurité européenne (policiers, gendarmes) ${ }^{32}$. Les acteurs qui nous intéressent présentent également le renforcement de la coopération judiciaire comme un élément essentiel en faveur de la légitimation du secteur européen de la sécurité. Le fait que la coopération

29. Ces relations ont été plusieurs fois évoquées au cours des entretiens.

30. Voir notamment Anne-Marie WAGNER, «Syndicalistes européens. Les conditions sociales et institutionnelles de l'internationalisation des militants syndicaux », Actes de la recherche en sciences sociales, $\mathrm{n}^{\circ}$ 155, 2004, p. 13-34. Également Olivier BAISNÉE, «En être ou pas. Les logiques de l'entre soi à Bruxelles », Actes de la recherche en sciences sociales, $\mathrm{n}^{\circ}$ 166-167, 2007, p. 110-121.

31. On retrouve, en l'espèce, les principales caractéristiques de «compromis culturel » mises en lumière par Marc Abélés et Irène Bélier dans leur étude des agents de la Commission européenne : Marc ABÉLÉS, Irène BÉLIER, «La Commission européenne : du compromis culturel à la culture politique du compromis », Revue française de science politique, vol.46: $\mathrm{n}^{\circ} 3,1996, \mathrm{p} .431-456$.

${ }^{32}$ Didier Bigo, Op. cit, 2005. 
policière se soit institutionnalisée avant l'entraide en matière judiciaire est souligné de façon systématique comme un déséquilibre nécessitant la mise en œuvre de dispositifs judiciaires afin d'encadrer l'action des forces de police.

Dans un autre registre, la totalité des protagonistes témoigne de l'apprentissage, au niveau européen, d'autres règles que celles inculquées lors de leur formation initiale. Dans cette perspective, le «relationnel », les « impératifs diplomatiques» et la nécessité de savoir « dépasser les pratiques nationales » sont érigés en valeurs partagées par tous, leur permettant de se distinguer nettement des acteurs restés dans les affaires nationales. Ces professionnels se définissent de façon collective comme des intermédiaires, au croisement des jeux juridiques nationaux et des processus transnationaux. Etroitement lié à cette définition qu'ils ont de leurs fonctions, ces magistrats et hauts fonctionnaires partagent et défendent une méthode qu'ils qualifient eux mêmes de «pragmatique » dans le but, selon eux, de favoriser une construction progressive et avant tout opérationnelle des dispositifs de coopération. Présentée comme la méthode européenne par excellence, cette démarche se différencie cependant de la méthode communautaire, car elle n'implique pas une intégration initiale au niveau des autorités communautaires. Dans ce type de configuration, les autorités nationales restent les principaux décideurs. Cette démarche dite «pragmatique» apparait, aux yeux des professionnels qui la défendent, comme l'unique moyen de renforcer la construction européenne dans un secteur aussi « sensible ${ }^{33}$ que celui de la justice pénale. Les expériences diplomatiques et politiques sont alors appréhendées comme un atout fondamental donnant une dimension supplémentaire au clivage politique/pratique.

On retrouve à travers ce positionnement, l'approche perçue comme classique du processus de construction européen qui consiste à favoriser la construction de dispositifs en fonction des cas concrets dans une démarche dite des «petit pas ». La dimension opérationnelle est ainsi promue au rang d'objectif principal par rapport à la cohérence juridique du dispositif. Cette approche s'appuie sur l'idée que la cohérence se construira inévitablement à travers l'expérience quotidienne. Dans cette perspective, l'exemple de Schengen est régulièrement cité en tant que référence. Ce n'est donc pas un hasard, si une partie des hauts fonctionnaires et magistrats qui ont contribué aux accords de Schengen, interviennent également dans la construction cognitive et juridique des instruments de coopération judiciaire tels qu'Eurojust et le mandat d'arrêt européen. Les logiques sociales des accords de Schengen trouvent ainsi un prolongement dans la mise en place d'un système européen judiciaire ${ }^{34}$. Le rejet des recommandations formulées par les mobilisations Corpus Juris et l'Appel de Genève en

33. Expression utilisée régulièrement dans les différents entretiens.

34. Pour une analyse du rôle des hauts fonctionnaires et de leurs pratiques lors des négociations des Accords de Schengen, voir : Virginie GUIRAUDON, «European integration and migration policy : vertical policy-making as venue shopping ", Journal of Common Market Studies, vol. 38, n² 2, 2000, p. 251-271. 
faveur d'un procureur européen s'inscrit dans ce système de pensées: "Les grands projets pour construire un espace pénal européen tout de suite ne sont pas en phase avec la méthode classique européenne. On ne peut pas communautariser tout de suite un secteur aussi sensible que le pénal, ce n'est pas réalisable politiquement ${ }^{35} »$.

Dans un tel cadre, la question de la souveraineté des États est utilisée comme un moyen de justifier le choix d'une approche permettant de construire un espace pénal européen tout en respectant la dimension intergouvernementale de ce domaine. Une telle démarche marque la mise à l'écart au niveau cognitif des autres solutions proposées dont celles en faveur d'une communautarisation générale et rapide du domaine pénal. Cette vision dominante de la construction européenne, soutenue par les experts du domaine, va influencer directement la manière dont Eurojust et le mandat d'arrêt européen vont être présentés par la suite comme des étapes nécessaires visant à renforcer l'entraide européenne dans sa dimension organisationnelle plutôt que dans le contenu des normes.

Ainsi dans le cas de l'unité Eurojust, cette approche a conduit à la mise en place d'une unité provisoire pro-Eurojust dès 2000 soit un an avant l'adoption définitive de la décision-cadre européenne stipulant la création de l'unité judiciaire. En choisissant de créer cette structure avant la définition et l'adoption de son cadre institutionnel et de sa base juridique, les représentants nationaux et les membres du SGCE à l'origine de cette initiative avait comme principale inquiétude de construire un dispositif qui trouve dans sa simple existence sa principale raison d'être : "Le fait que pro-Eurojust existe est déjà une avancée en soit. Le plus important dans la construction européenne est que le dispositif existe. Ensuite vient la question de sa forme et de son activité ${ }^{36} »$. Dans cette logique d'action, la définition des bases légales concernant les principes généraux du droit et les procédures judiciaires n'intervient qu'a posteriori afin de justifier et de légitimer ce qui existe déjà. Une telle dynamique de production n'est pas sans conséquence sur la manière dont Eurojust et son activité vont se développer par la suite dans une recherche quotidienne de légitimité vis-à-vis des autres autorités européennes et nationales (Mégie, 2007).

Dans le cas du mandat d'arrêt européen, cette même démarche va être utilisée dans un contexte politique particulier soit quelques jours après les attentats du 11 septembre. Au nom de la rapidité et de l' «efficacité politique ${ }^{37}$ » la décision-cadre finale va subir une forte reformulation juridique. La réécriture de la décision-cadre est effectuée en petit comité, 3 à 4 personnes du SGCE et de la Chancellerie belge, dans une stratégie diplomatique visant à favoriser avant tout une adoption rapide

\footnotetext{
35. Entretien réalisé avec un haut fonctionnaire du ministère de la Justice belge, février 2003.

36. Entretien réalisé avec un magistrat français travaillant dans la coopération judiciaire, septembre 2001. 37. Ibid.
} 
par les États, quitte à écarter certains pans du projet de la Commission européenne, notamment concernant les droits de la défense.

«Nous devions être réalistes, les États s'étaient mis d'accord pour une adoption, mais ils n'allaient quand même pas tout laisser passer sans négociation. L'idée était de poser un premier cadre accepté par tous, pour ensuite consolider l'ensemble du dispositif et le rendre le plus efficace possible étant donné les différences nationales ${ }^{38} "$.

La rapidité des négociations ainsi que le positionnement des acteurs au cœur de la formulation juridique de la décision-cadre, n'est pas sans répercussion sur la forme que va prendre le dispositif d'extradition. Pour certains membres du SGCE ou des autorités nationales, défendant l'idée d'une évolution pragmatique, le texte de Laeken aura eu comme intérêt essentiel de dépasser la logique du plus petit dénominateur commun.

L'imposition d'une vision opérationnelle de la coopération judiciaire européenne doit donc être comprise comme le produit d'une spécialisation européenne de certains professionnels. Le partage d'une culture de compromis tournée vers une approche pragmatique dans un contexte largement dominé par les questions de sécurité permet d'expliquer comment et pourquoi la justice au niveau européen a été fortement influencée par les questions de police et de sécurité.

$\mathrm{Au}$ cœur du jeu institutionnel européen ${ }^{39}$ en tant que gestionnaire de l'agenda, rédacteur et promoteur des solutions d'action publique, l'importance de ces acteurs membres des autorités exécutives symbolise la centralité de ces dernières dans la production européennes des normes pénales et la relative relégation des autorités législatives et judiciaires.

Cependant s'arrêter à ce stade de l'analyse ne permet pas de comprendre l'ensemble du processus de fabrication des normes pénales européennes. Comme nous l'avons montré, il est essentiel de considérer la phase de mise en œuvre des dispositifs judiciaires à travers leurs reformulations

38. Entretien réalisé avec un membre du SGCE, avril 2004.

39. Jacques DE MAILLARD, Eve FOUILLEUX, Andy SMITH, « Technical or political ? : the working groups of the EU Council of Ministers », Journal of European Public Policy, vol. 12, n 4, 2005, p. 609-623. 
juridiques et institutionnelles ${ }^{40}$. Encore largement en cours, ces phases de reformulation juridique marquent l'investissement d'un autre type d'acteurs du droit. Fortement relégués durant les négociations diplomatiques, les cours constitutionnels et les magistrats nationaux jouent un rôle primordial dans la redéfinition des dispositifs en tant que praticiens du droit ${ }^{41}$. Dans cette perspective, une analyse générale des interactions entre ces praticiens et les professionnels que nous venons d'étudier représente une piste de recherche primordial afin d'interroger la participation des professionnels du droit à la gouvernementalité européenne ${ }^{42}$.

\section{Bibliographie}

Marc ABÉLÉS, Irène BÉLIER, «La Commission européenne : du compromis culturel à la culture politique du compromis », Revue française de science politique, vol.46: ${ }^{\circ} 3$, 1996, p. 431-456.

Olivier BAISNÉE, «En être ou pas. Les logiques de l'entre soi à Bruxelles », Actes de la recherche en sciences sociales, $\mathrm{n}^{\circ} 166-167,2007$, p. 110-121.

Howard BECKER, Outsiders, The Free Press of Glencoe, 1963, Trad. Paris, Editions Métailié, Paris, 1985.

Didier BIGO, «La mondialisation de l'(in)sécurité ? Réflexions sur le champ des professionnels de la gestion des inquiétudes et analytique de la transnationalisation des processus d'(in)sécurisation », Cultures \& Conflits, $\mathrm{n}^{\circ}$ 58, 2005, p. 53-100.

Didier BIGO, Polices en réseaux, Presses de Sciences Po, 1996.

40. Antoine MEGIE, Eurojust et le mandat d'arrêt européen. L'européanisation du pouvoir judiciaire : genèse, luttes et enjeux de la construction de deux dispositifs de coopération pénale, Thèse de Doctorat de science politique, sous la direction de Renaud Dehousse, Institut d'Etudes Politiques de Paris, novembre 2007.

${ }^{41}$. Pour une étude de la mise en œuvre et du fonctionnement de l'unité Eurojust : Antoine MEGIE, «L'institutionnalisation d'un pouvoir judiciaire européen incertain en quête de légitimité : l'unité de coopération Eurojust », Politique Européenne, ${ }^{\circ}$ 24, Paris, l'Harmattan, 2008 ; Pour le mandat d'arrêt européen, voir : Maik MARTIN, "Franchir l'infranchissable ? Coopération judiciaire et reconnaissance mutuelle dans un espace européen de justice, liberté, et sécurité », Cultures et Conflits, Paris, l'Harmattan, nº62, juin 2006, p 63-77.

42. Antoine VAUCHEZ, "The Force of a Weak Field: Law and Lawyers in the Government of the European Union (For a Renewed Research Agenda) », International Political Sociology, Blackwell Publishing, vol. 2, n², juin 2008, p. 128-144; Antonin COHEN, Julien WEISBEN, «Laboratoires du constitutionnalisme européen. Expertises académiques et mobilisations politiques dans la promotion d'une Constitution européenne », Droit et société, $\mathrm{n}^{\circ} 60,2005$, p. 353-371. 
Paul BOUFFARTIGUE, «Introduction », in Yvette LUCAS, Claude DUBAR (dir.), Genèse et dynamique des groupes professionnels, Lille, Presses Universitaires de Lille, 1994, p. 327-339.

Antonin COHEN, Antoine VAUCHEZ (dir.), La Constitution européenne : élites, mobilisations, votes, Bruxelles, éditions de l’Université de Bruxelles, 2007, p. 271-291.

Antonin COHEN, Yves DEZALAY, Dominique MARCHETTI, «Introduction: Esprits d'État, entrepreneurs d'Europe », Actes de la recherche en sciences sociales, $\mathrm{n}^{\circ}$ 166-167, 2007, p. 4-13.

Antonin COHEN, Julien WEISBEN, «Laboratoires du constitutionnalisme européen. Expertises académiques et mobilisations politiques dans la promotion d'une Constitution européenne ", Droit et société, $\mathrm{n}^{\circ} 60,2005$, p. 353-371.

Jacques COMMAILLE, Martine KALUSZYNSKI (dir.), La fonction politique de la justice, Paris, La Découverte, 2007.

Wanda De LEMOS DE CAPPELER, «La transnationalisation du champ pénal : réflexions sur les mutations de crise et de contrôle », Droit et société, n 35, 1997, p. 61-77.

Jacques DE MAILLARD, Eve FOUILLEUX, Andy SMITH, « Technical or political ? : the working groups of the EU Council of Ministers ", Journal of European Public Policy, vol. 12, ${ }^{\circ} 4,2005$, p. $609-623$.

Yves DEZALAY, «De la défense de l'environnement au développement durable. L'émergence d'un champ d'expertise des politiques européennes ", Actes de la recherche en sciences sociales, 2007, p. 68 .

Yves DEZALAY, Garth BRYANT, The Internationalization of Palace Wars: Lawyers, Economists, and the Contest to Transform Latin American States, Chicago, University Press Book, 2002.

Laurence DUMOULIN, « De l'impact des experts judiciaires sur la fabrique de la justice: d'une justice imposée à une justice négociée ? », dans Laurence DUMOULIN, Stéphane LA BRANCHE, Cécile ROBERT, Philippe WARIN (dir), Le recours aux experts : raisons et usages politiques, Grenoble, Presses universitaires de Grenoble, coll. « Symposium », 2005.

Didier GEORGAKAKIS (dir), Les Métiers de l'Europe politique : acteurs et professionnalisations de l'Union européenne, Strasbourg, Presses universitaires de Strasbourg, 2002.

Virginie GUIRAUDON, «European integration and migration policy: vertical policy-making as venue shopping », Journal of Common Market Studies, vol. 38, n² 2, 2000, p. 251-271. 
Azilis MAGUER, Les frontières intérieures Schengen: Dilemmes et stratégies de la coopération policière et douanière franco-allemande, Max Planck Institut, éd. Iuscrim, 2004.

Michel MANGENOT, Hélène MICHEL, Natacha PARIS, Une Europe de la justice : mobilisation contre la "criminalité organisée" et institutionnalisation d'un espace judiciaire pénal européen (19962001), Paris, Mission de recherche Droit et Justice, 2004.

Maik MARTIN, «Franchir l'infranchissable ? Coopération judiciaire et reconnaissance mutuelle dans un espace européen de justice, liberté, et sécurité », Cultures et Conflits, Paris, l'Harmattan, $\mathrm{n}^{\circ} 62$, juin 2006, p 63-77.

Antoine MEGIE (dir), "Arrêter et juger en Europe : genèse, luttes et enjeux de la coopération pénale », Cultures et Conflits, ${ }^{\circ} 62$, Paris, l'Harmattan, Printemps 2006.

Antoine MEGIE, Eurojust et le mandat d'arrêt européen. L'européanisation du pouvoir judiciaire: genèse, luttes et enjeux de la construction de deux dispositifs de coopération pénale, Thèse de Doctorat de science politique, sous la direction de Renaud Dehousse, Institut d'Etudes Politiques de Paris, novembre 2007.

Antoine MEGIE, «L'institutionnalisation d'un pouvoir judiciaire européen incertain en quête de légitimité : l'unité de coopération Eurojust », Politique Européenne, n² 24, Paris, 1'Harmattan, 2008.

Violaine ROUSSEL, «Les magistrats français, des cause lawyers malgré eux ?», Politix, vol. 16, $\mathrm{n}^{\circ}$ 62, 2003, p. 93-113.

Guillaume SACRISTE, Antoine VAUCHEZ, «Les «bons offices» du droit international: la constitution d'une autorité non politique dans le concert diplomatique des années 1920 », Critique internationale, Paris, $\mathrm{n}^{\circ} 25,2005$, p. 101-117.

Yves SUREL, «L'intégration européenne vue par l'approche cognitive et normative des politiques publiques », Revue française de science politique, vol. 50, $\mathrm{n}^{\circ}$ 2, avril 2000, p. 241.

Antoine VAUCHEZ, L'institution judiciaire remotivée : le processus d'institutionnalisation d'une "nouvelle justice" en Italie : 1960-2000, Paris, LGDJ, coll. « Droit et Société », 2004.

Antoine VAUCHEZ, «The Force of a Weak Field: Law and Lawyers in the Government of the European Union (For a Renewed Research Agenda) », International Political Sociology, Blackwell Publishing, vol. 2, n², juin 2008, p. 128-144.

Anne-Marie WAGNER, «Syndicalistes européens. Les conditions sociales et institutionnelles de l'internationalisation des militants syndicaux », Actes de la recherche en sciences sociales, $\mathrm{n}^{\circ} 155$, 2004, p. 13-34. 
